\title{
Tracking Control System Using an Incident Radiation Angle Microsensor
}

José M. Quero, Senior Member, IEEE, Carmen Aracil, Leopoldo G. Franquelo, Fellow, IEEE, Jordi Ricart, Pablo R. Ortega, Manuel Domínguez, Member, IEEE, Luis M. Castañer, Senior Member, IEEE, and Rafael Osuna

\begin{abstract}
For some industrial applications, an accurate estimation of a light source position is needed. That is the case for a heliostat, a device that projects sunlight onto a focus hundreds of meters away from its aiming point. In this paper, we present a novel sensor design for generating an alignment error signal. Included is a detailed study of its response, which shows that certain geometrical design parameters are necessary to achieve desired accuracy. This sensor has been implemented using microelectromechanical system techniques to achieve a robust structure at low cost and it has been successfully applied to sun-tracking systems. Experimental results obtained in field tests are included.
\end{abstract}

Index Terms-Microelectromechanical system (MEMS), microsensors, power generation, solar energy, sun-tracking control.

\section{INTRODUCTION}

$\mathbf{T}$ HE USE of renewable energy is fundamental to achieving sustainable development. Specific technologies have been intensively studied during the last decade [1], [2]. These technologies are now sufficiently mature to be applicable, but in most cases, they are not competitive when compared to conventional energy sources [3]. For that reason, renewable energy plant managers are striving to reduce the manufacturing, installation, and maintenance costs of their plants.

In the case of solar power plants, the performance of these installations strongly depends on the ability of the solar power receivers to track the sun.

Several methods have been proposed for large-scale conversion of solar energy to electricity. On the one hand, there are systems made up of devices that directly transform the sun radiation into electricity, e.g., conventional photovoltaic (PV) or high-concentration PV plants [4], [5]. The surface of these devices must be perpendicular to the sun radiation to achieve maximum performance [6]; therefore, direct tracking control should be implemented.

On the other hand, there are solar-concentrating power plants, like the one depicted in Fig. 1. Large mirrors comprising

Manuscript received April 3, 2006; revised September 9, 2006. Abstract published on the Internet January 27, 2007. This work was supported by the Spanish Ministerio de Ciencia y Tecnología under Grant TIC2000-0344-P4-0.

J. M. Quero, C. Aracil, and L. G. Franquelo are with the Department of Electronic Engineering, University of Seville, 41092 Seville, Spain (e-mail: quero@us.es).

J. Ricart, P. R. Ortega, M. Domínguez, and L. M. Castañer are with the Departamento de Ingeniería Electrónica de la Escuela Técnica Superior de Ingeniería de Telecomunicación, Universitat Politècnica de Catalunya, 08034 Barcelona, Spain.

R. Osuna is with Sanlúcar Solar Solúcar, 41018 Seville, Spain.

Color versions of one or more of the figures in this paper are available online at http://ieeexplore.ieee.org.

Digital Object Identifier 10.1109/TIE.2007.893075

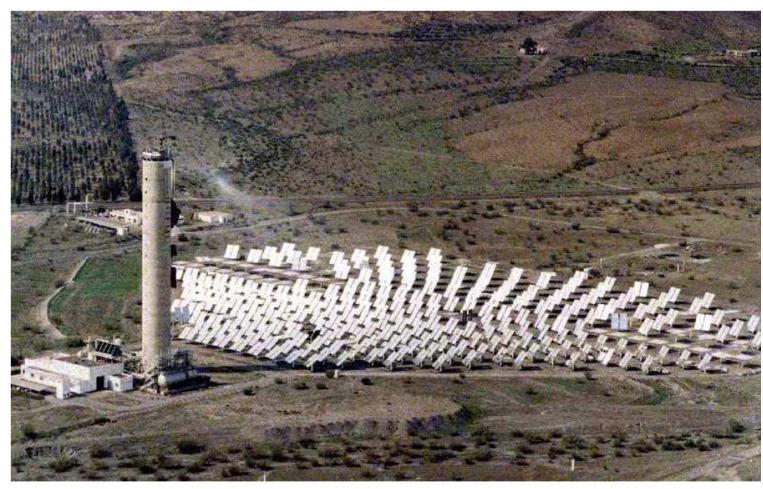

Fig. 1. Solar-concentrating power plant in Almería (Spain).

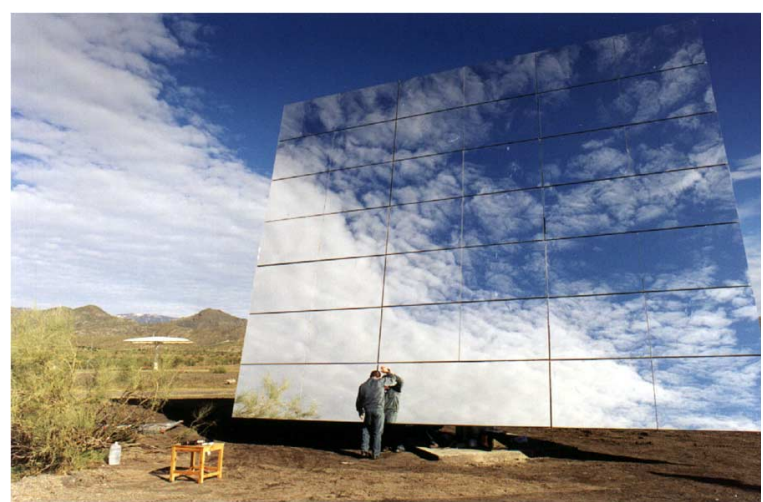

Fig. 2. Mirror used in solar-concentrating power plants.

several facets track the sun's trajectory to reflect its light onto a central absorber. These mirrors, which are called heliostats, are heavy supporting structures with large reflective surfaces, like the one shown in Fig. 2. These devices rotate with respect to vertical and horizontal axes, namely, azimuth and elevation axes, respectively. The sunlight is projected onto a specific area within the absorber that is hundreds of meters away from the mirror. As a maximum projection error of one quarter of meter is allowable, the positioning angle error must be no more than $1 \mathrm{mrad}$. In this application, accurate reflective tracking control is needed to guarantee the correct positioning of the ray reflected onto its aiming point in the receiver.

Some other applications also require an accurate estimation of the position of an object [7]. For instance, in the absence of the magnetic field of the earth (e.g., in satellite navigation), the position of an object is defined with respect to the position of the sun [8]. 
Traditionally, control mechanisms implemented in these tracking devices follow an open-loop scheme because of the lack of sun sensors accurate enough to precisely determine the sun's position, or because of the high cost of such sensors. However, this open-loop control needs to minimize the influence of a series of mechanical error sources:

1) mechanical manufacturing tolerances;

2) mechanical structure deformations;

3) accuracy of the encoders located in the rotation axes;

4) relative positioning errors of the heliostats with respect to the absorber.

To reduce these errors, special care should be taken during installation. Periodical maintenance updating error estimation algorithms have also been proposed [9].

Some sensors' structures have been described in the literature and most of them are based on a lens and a quadrant cell solar detector [10]. The sun sensor lens projects an image of the sun onto the detector and produces current in the photodiodes. Although this solution is technically valid, it is not economically feasible because of the size of such a sensor and the difficulties to achieve the precision needed during its fabrication. An attempt to increase the integration of this sensor is suggested in [11] by using a lateral photodiode [12]. In this solution, the carriers generated by the photoelectric effect in the body of the diodes are divided into two currents that are inversely proportional to the distance of the incident ray to the output electrodes. However, when the incident angle is near the center of the sensing surface, it lacks sensitivity.

An alternative to these conventional sensors-a chargecoupled device sensor-has been suggested in [13], but complex data processing is necessary to obtain high accuracy.

To circumvent all these problems, we present a novel control scheme using a low-cost sun-tracking sensor. All previous implementations were based on macrorealizations, which are expensive to manufacture and difficult to adjust. The sensor proposed here is manufactured using microelectromechanical system (MEMS) technologies. The use of this technology reduces the manufacturing costs of the sensor and significantly increases its accuracy. The proposed structure has a geometrical form factor that inherently provides an amplification of the incident angle signal. Moreover, its easy manufacturing process reduces its cost and makes it suitable for all the applications mentioned previously.

This paper is organized as follows: Section II describes the sensor that has been designed using MEMS technologies, including the device modeling and realization. In Section III, a complete control algorithm using this sensor is presented. The application of this controller is detailed in Section IV, and its use in heliostats is discussed, including experimental results. Finally, in Section V, the main conclusions are summarized.

\section{INCIDENT ANGLE MicRosensor}

\section{A. Sensor Principle}

The proposed sensor consists of two photodiodes partially covered with a metal shield that has an aperture (see Fig. 3). When a light ray falls on the sensor, only a fraction of the area

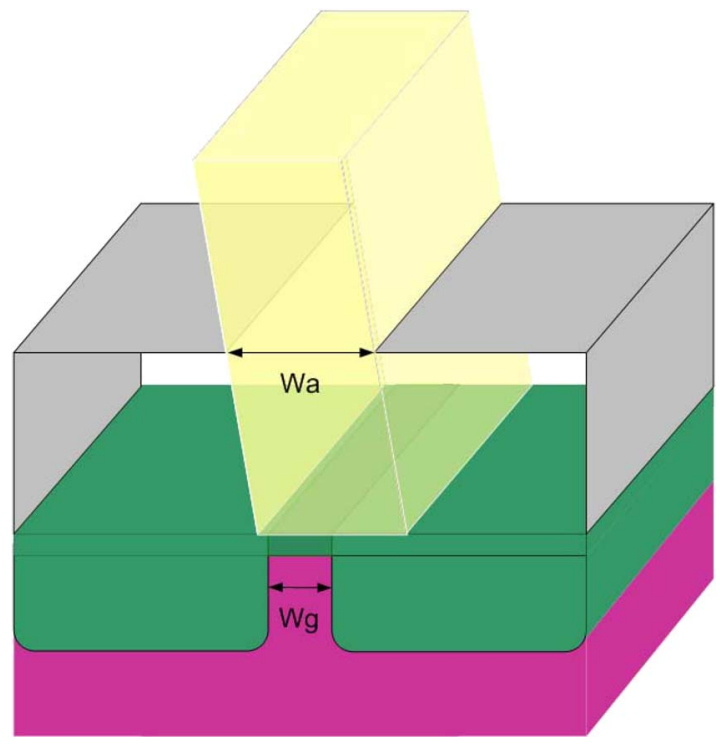

Fig. 3. Three-dimensional view of the sensor with a deviation in the incident angle.

in each photodiode is illuminated. The incident light energy generates a photocurrent that is proportional to the illuminated area in each photodiode. This area depends on the shadow projected over the photodiodes, and therefore on the incident angle of the light ray. The gap between the photodiodes prevents the combination of carriers, and each device acts as a sink for its generated charge, thus significantly improving the sensitivity of the device in comparison to a continuous lateral photodiode.

Another alternative design involves the use of a metal shield to project shadow instead of an aperture. In this case, the operation principle of the sensor is the same and the previous analysis is valid. However, this negative approach has the inconvenience of being more sensitive to indirect radiation.

\section{B. Device Modeling}

The calculus of the current generated by the PV effect has been widely studied [14]. The basic parameter that expresses the resulting efficiency of a photodiode is the spectral response $S_{R}$, which is defined as the ratio of the number of carriers generated to the number of incident photons with wavelength $\lambda$. The current density generated $J_{L}(\lambda)$ is determined by

$$
J_{L}(\lambda)=q N_{F}(\lambda)(1-R) S_{R}(\lambda)
$$

where $q$ is the electron charge, $N_{F}(\lambda)$ is the number of incident photons per unity area and time, and $R$ is the photon reflection on the semiconductor surface. $S_{R}$ can be deduce by integrating the continuity equation and obtain an expression of the minority carrier concentrations in each region of the photodiode. The total photocurrent can be calculated by integrating that expression over the entire spectral range, i.e.,

$$
I_{L}=A \int_{0}^{\lambda_{g}} J_{L}(\lambda) d \lambda=q A \int_{0}^{\lambda_{g}} N_{F}(\lambda)(1-R) S_{R}(\lambda) d \lambda
$$




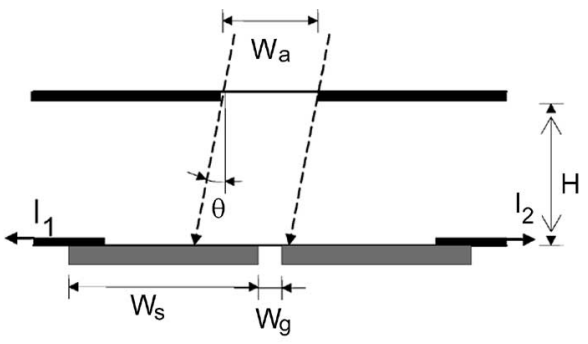

(a)

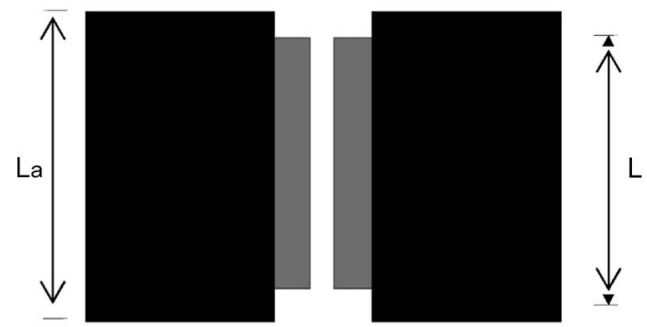

(b)

Fig. 4. Schematic representation of the sensor with a deviation in the incident angle. (a) Front view. (b) Top view.

where $A$ is the total illuminated area in the photodiode. Notice that the photocurrent generated is proportional to the incident radiation.

The behavior of the proposed microsensor is determined by the projection of the shadow onto the photodiodes. When the sensor is pointing at the light ray, this projection is perpendicular, and therefore, the illuminated areas in the photodiodes are equal. As the photocurrent generated in each photodiode is proportional to its illuminated area, their current difference is null. When the ray is not perpendicular, there is a deviation in the incident angle $\theta$, as depicted in Fig. 4(a) and (b). We can determine the angle of the incident ray by measuring the difference between the photocurrents generated.

Let us define the photodiode dimensions $L$ and $W_{s}$, the separation between the photodiodes $W_{g}$, the aperture size in the shield $W_{a}$, and the distance from the cover to the photodiode $H$. The illuminated area in each photodiode is given by

$$
\begin{aligned}
& A_{1}=\left(\frac{W_{a}}{2}-\frac{W_{g}}{2}+H \tan \theta\right) \cdot L \\
& A_{2}=\left(\frac{W_{a}}{2}-\frac{W_{g}}{2}-H \tan \theta\right) \cdot L .
\end{aligned}
$$

Using (2), the difference between the photocurrents generated is given by

$$
I_{1}-I_{2}=2 \cdot H \cdot L \cdot \tan \theta \cdot J_{L}
$$

The incident angle $\theta$ can be obtained from (5) as

$$
\theta=\arctan \left(\frac{I_{1}-I_{2}}{2 \cdot H \cdot L \cdot J_{L}}\right)
$$

If we consider a given $\theta$, the current difference increases when using larger $L$ and $H$. Unfortunately, $I_{1}-I_{2}$ also depends on the current density and therefore on the total radiation. For this reason, if there is a reduction in the direct sun radiation, e.g., a cloud crossing, it will decrease the error signal. To avoid the interdependence between $J_{L}$ and the angle $\theta$, we will consider normalizing the current difference.

Let us consider the addition of the following generated currents:

$$
I_{1}+I_{2}=L \cdot\left(W_{a}-W_{g}\right) \cdot J_{L}
$$

It is worth noting that $I_{1}+I_{2}$ does not depend on the incident angle but is proportional to $J_{L}$ and therefore to the direct radiation. This magnitude can be used to normalize the current difference and make it independent of the direct radiation. If we define the form factor gain $G_{F}$ as

$$
G_{F}=\frac{H}{W_{a}-W_{g}}
$$

and divide (5) by (7), we obtain a simple expression for the error angle $\theta$. That is,

$$
\theta=\arctan \left(\frac{1}{2 G_{F}} \frac{I_{1}-I_{2}}{I_{1}+I_{2}}\right) .
$$

For small deviations, the current difference $I_{1}-I_{2}$ becomes proportional to $\theta$. That is,

$$
\frac{I_{1}-I_{2}}{I_{1}+I_{2}}=2 G_{F} \tan \theta \cong 2 G_{F} \theta .
$$

Notice that $G_{F}$ can be regarded as a geometric amplification factor, because with a larger $G_{F}$, we obtain a larger error signal.

The current difference is a continuous increasing function of the incident angle until a maximum deviation error occurs when the incident ray reaches the edge of one of the photodiodes. In this situation, one of the photocurrents becomes zero and then

$$
\theta_{\max }=\arctan \left(\frac{1}{2 G_{F}}\right) .
$$

In this expression, it is clear that the maximum incident angle value decreases when $G_{F}$ increases. There is a tradeoff between the sensitivity of the sensor and the maximum deviation angle that can be measured. On one hand, an increase in $G_{F}$ provides a larger sensing range, but on the other hand, it also represents less accuracy. For this reason, the selection of the form factor $G_{F}$ depends on the application to balance its accuracy and range.

Normally, $W_{s}$ and $L$ are designed so that their maximum admissible value has a large output current difference. $W_{g}$ is minimized provided its value guarantees isolation between the photodiodes. $W_{a}$ is also designed to define the largest possible window taking into account the photodiode limits. $H$ is the only parameter that the user can determine to obtain a desired sensitivity or dynamic range. 


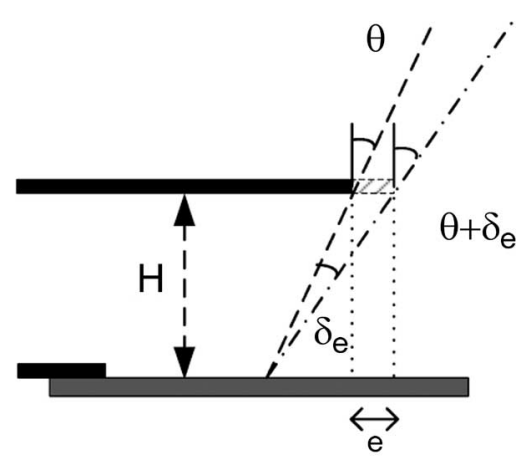

Fig. 5. Error angle $\delta_{e}$ when a deviation $e$ occurs during the alignment of the shield with respect to the photosensors.

The previous analysis is based on the hypothesis that the shield is perfectly aligned with respect to the photodiodes and shield. If this is not the case, there is an error angle $\delta_{e}$ generated by a misalignment $e$, as it is shown in Fig. 5. It can be deduced that $\delta_{e}$ is related to $e$ by

$$
\delta_{e}=\arctan \left(\frac{\frac{e}{H} \cos ^{2} \theta}{1-\frac{e}{H} \cos \theta \sin \theta}\right) .
$$

The maximum error angle corresponds to $e / H$ for $\theta=0$, but with a specific manufacturing MEMS alignment process, $e$ is several orders of magnitude lower than $H$. For special applications where this deviation error $\delta_{e}$ is not tolerated, it can be compensated with an offset adjustment in the electronic conditioning circuitry or using a closed-loop scheme, as will be explained in Section IV.

\section{Sensor Topologies}

1) Four Quadrant Sensor: In this scheme, four photodiodes are covered with a common shield. A metal shield was manufactured by patterning a metal layer on a Pyrex wafer. Finally, this shield was glue bonded onto the photodiodes. Fig. 6 shows a top schematic representation of the sensor. The photocurrents have to be added in pairs to obtain an error signal corresponding to the angular deviation in one axis. For instance, according to (6), the incident angle with respect to axis $A_{1}$ is given by

$$
\theta=\arctan \left(\frac{\left(I_{a}+I_{b}\right)-\left(I_{c}+I_{d}\right)}{2 \cdot H \cdot L \cdot J_{L}}\right) .
$$

In this way, the influence of the deviation in another axis is cancelled out.

2) Two Independent Axes Sensor: In this scheme, two modules are arranged in perpendicular axes to determine the incident angle, as depicted in Fig. 7. Each differential sensor is independent from the other, but $L_{a}$ should be large enough to guarantee that a misalignment on one axis does not affect the active area of the other sensor. Special care should be taken in the layout of both sensors so as to avoid any interference between them.

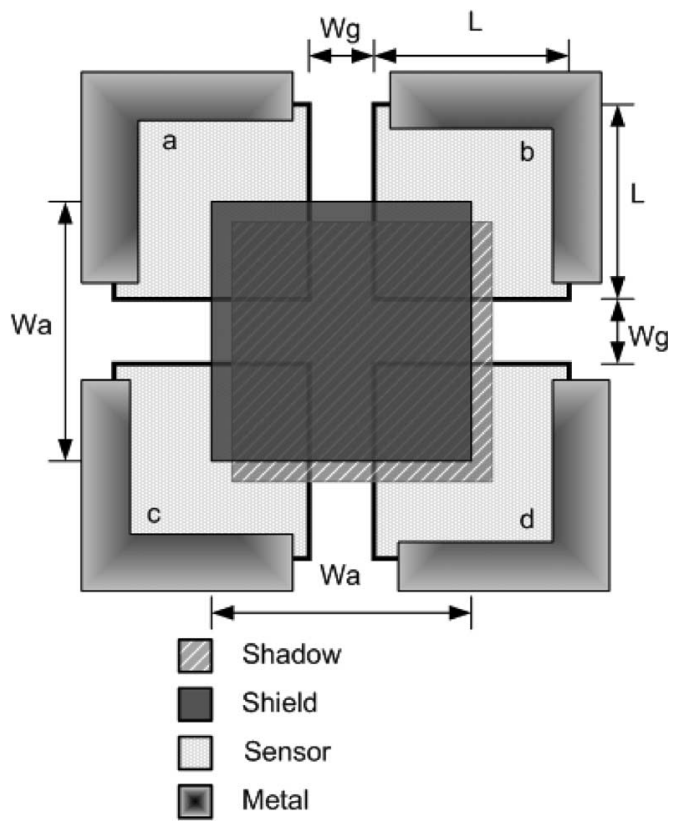

Fig. 6. Four quadrant sensor layout.

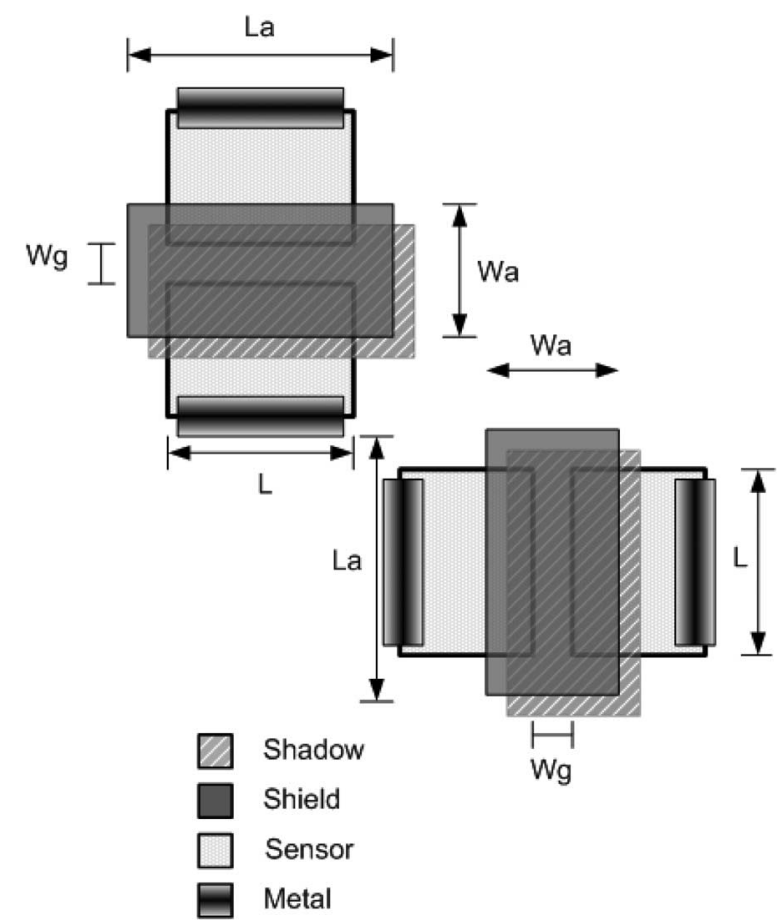

Fig. 7. Two independent axes sensor layout.

\section{Sensor Implementation}

Four quadrant sensor and two independent axes sensor designs were implemented in order to compare their performances. To achieve the desired static response of the sensors previously defined by the applications, their geometrical parameters were calculated. Their resulting values are presented in Table I.

The sensor responses have been experimentally verified under different working conditions for all implementations. A picture of four quadrant sensors in positive and negative 
TABLE I

DESIGN PARAMETERS

\begin{tabular}{|c|c|c|c|}
\hline & $\begin{array}{c}\text { Four } \\
\text { quadrant }\end{array}$ & $\begin{array}{c}\text { Two } \\
\text { independent } \\
\text { axes (high } \\
\text { sensitivity) }\end{array}$ & $\begin{array}{c}\text { Two } \\
\text { independent } \\
\text { axes (low } \\
\text { sensitivity) }\end{array}$ \\
\hline $\mathrm{Wg}$ & $200 \mu \mathrm{m}$ & $150 \mu \mathrm{m}$ & $150 \mu \mathrm{m}$ \\
\hline $\mathrm{Wa}$ & $3500 \mu \mathrm{m}$ & $1800 \mu \mathrm{m}$ & $1800 \mu \mathrm{m}$ \\
\hline $\mathrm{Ws}$ & $2000 \mu \mathrm{m}$ & $3600 \mu \mathrm{m}$ & $3600 \mu \mathrm{m}$ \\
\hline $\mathrm{L}$ & $2000 \mu \mathrm{m}$ & $4000 \mu \mathrm{m}$ & $4000 \mu \mathrm{m}$ \\
\hline $\mathrm{H}$ & $2000 \mu \mathrm{m}$ & $2000 \mu \mathrm{m}$ & $500 \mu \mathrm{m}$ \\
\hline
\end{tabular}

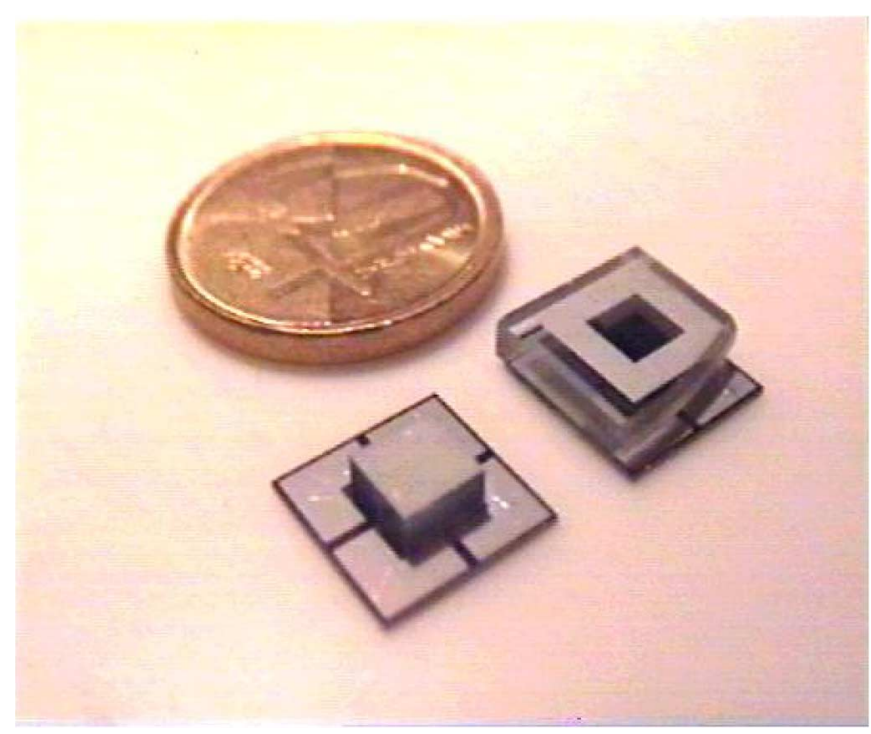

Fig. 8. Four quadrant positive and negative sensors.

(a)

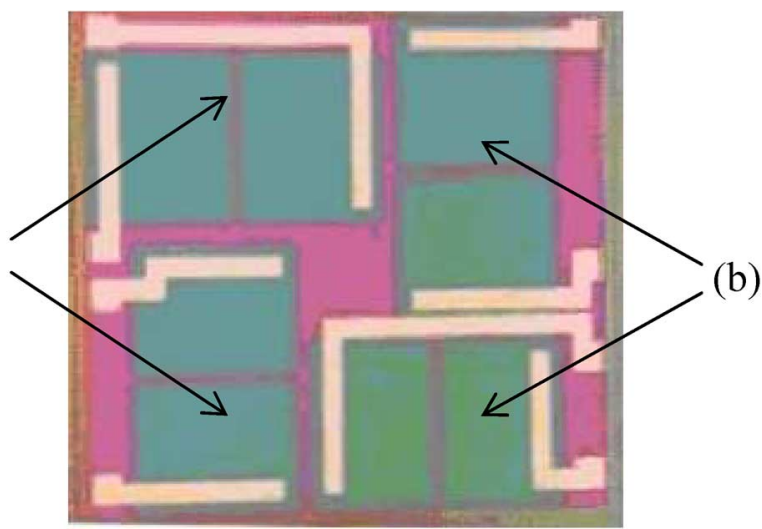

Fig. 9. Die with the photodiodes. (a) Sensors used for fine-tuning. (b) Sensors used for wide range.

schemes is presented in Fig. 8. The main drawback of the four quadrant topology is that the resulting error signals are coupled, and therefore, a more complicated control strategy is needed.

Two independent differential modules in each axis comprise the final version of the sensor used in the next application. The only design difference between the modules of the same axis is the use of different $H$ values. This way one sensor has greater sensitivity and the other has wider dynamic range. A photograph of the sensor die without the cover is shown in Fig. 9, where we see in Fig. 9(a) sensors used for fine-tuning

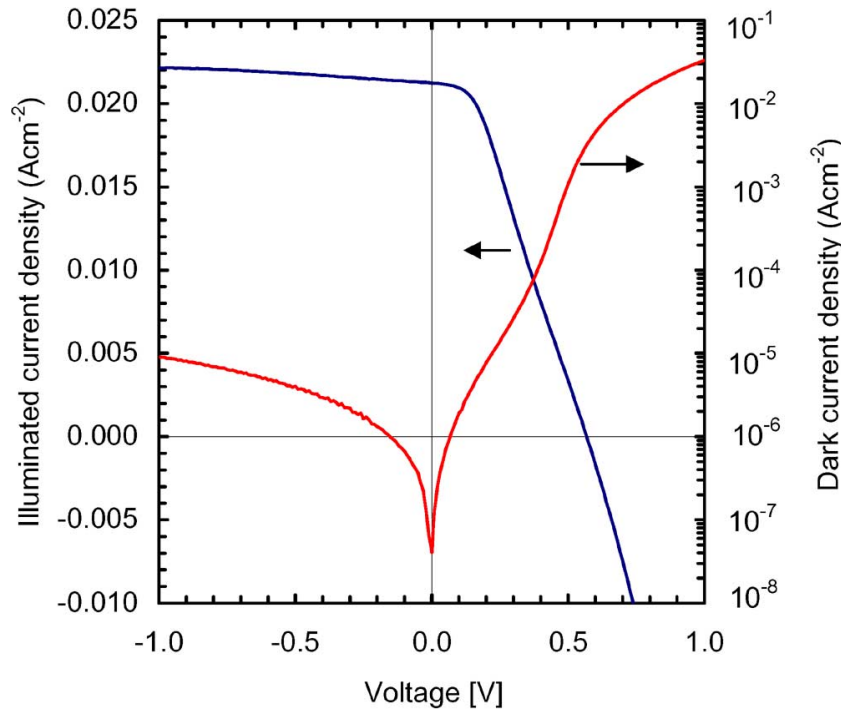

Fig. 10. Illuminated and dark current versus voltage of a photodiode from the two independent axes sensor.

and in Fig. 9(b) sensors used for wide range detection by using $H=2000 \mu \mathrm{m}$ and $H=500 \mu \mathrm{m}$, respectively.

Photocells were characterized in laboratory tests using a Solar Simulator, and the illuminated and dark currents generated and measured are plotted in Fig. 10.

A controller board was designed, which included three blocks, namely: 1) a signal conditioning circuit; 2) a microcontroller; and 3) an H-Bridge that uses relays for the actuation of the geared dc motors installed in the mechanical structures. The board design was based in a distributed network control system for industrial plant automation [15]. The signal conditioning circuit includes a polarization for adding and subtracting circuits (Fig. 11). These circuits are replicated for every differential module. Differential and instrumentation amplifiers are used in this design. Their gain can be adjusted with a potentiometer to obtain the desired accuracy in the error signal. In addition, other potentiometers are included for offset voltage compensation. These offset potentiometers are also useful to compensate for any geometrical deviation during the sensor installation.

The resulting transfer functions of these circuits are as follows:

$$
\begin{aligned}
V_{o} & =k_{2} \frac{R_{\mathrm{POT} 1}}{2}\left(I_{D 2}-I_{D 1}\right)+V_{\text {ref }} \\
k_{2} & =5+\frac{200 k}{R_{\mathrm{POT} 2}} \\
V_{o} & =2 k_{1} V_{\mathrm{POL}}-k_{1} \frac{R_{\mathrm{POT} 1}}{2}\left(I_{D 2}+I_{D 1}\right) \\
k_{1} & =\frac{R_{\mathrm{POT} 3} \| R_{\mathrm{in}}}{R_{\mathrm{POT} 3} \| R_{\text {in }}+R_{\text {in }}} \frac{R_{\mathrm{in}-}+R_{F}}{R_{\mathrm{in}-}} .
\end{aligned}
$$

The response of the sensor exposed to direct sunlight is presented in Fig. 12. This oscillogram was captured at noon, which is why the output voltage of the elevation axis voltage is almost constant, whereas that of the azimuth varies according 

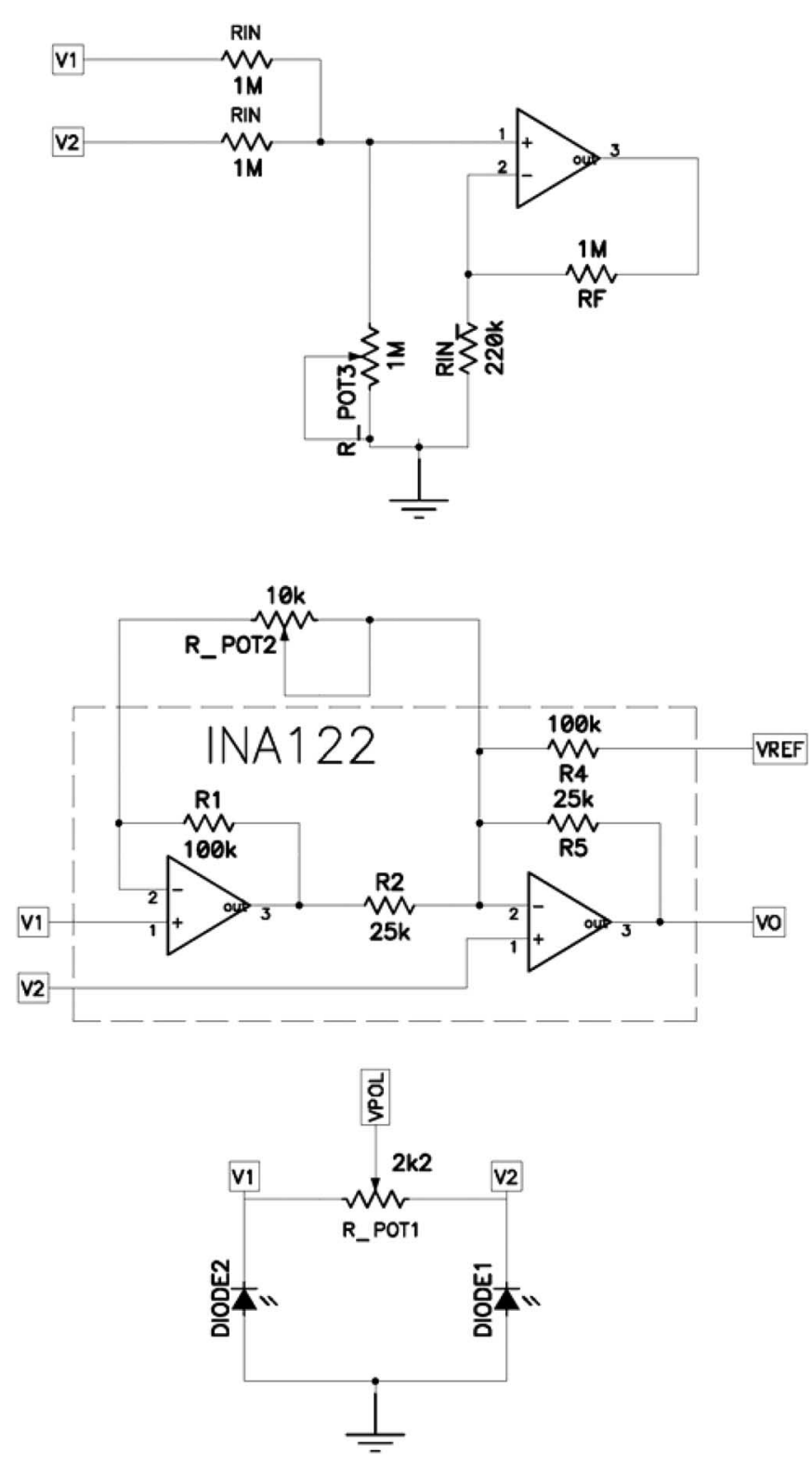

Fig. 11. Polarization and conditioning circuits for the subtraction and addition of photocurrents.

to sun movement. Notice that the azimuth voltage decreases more than $100 \mathrm{mV}$ in $22.5 \mathrm{~s}$, which approximately represents a milliradian displacement of the sun, thus providing a sufficient signal resolution for an accurate angle positioning system.

The sensor outputs are digitalized and filtered using an MCORE MMC2107 microcontroller, which will also implement the control algorithm. The sample rate is $1 \mathrm{kHz}$ with 10-bit resolution. A low-band 75th-order digital filter FIR was used, with a bandstop 0.005 of Nyquist frequency. An example of the filter response is shown in Fig. 13.

\section{Control System}

The sensor implemented allows high-quality error signals for sun tracking when the sun radiation is above a certain minimum value; however, there are other situations (for example, when there are clouds) in which specific control actuation has to be

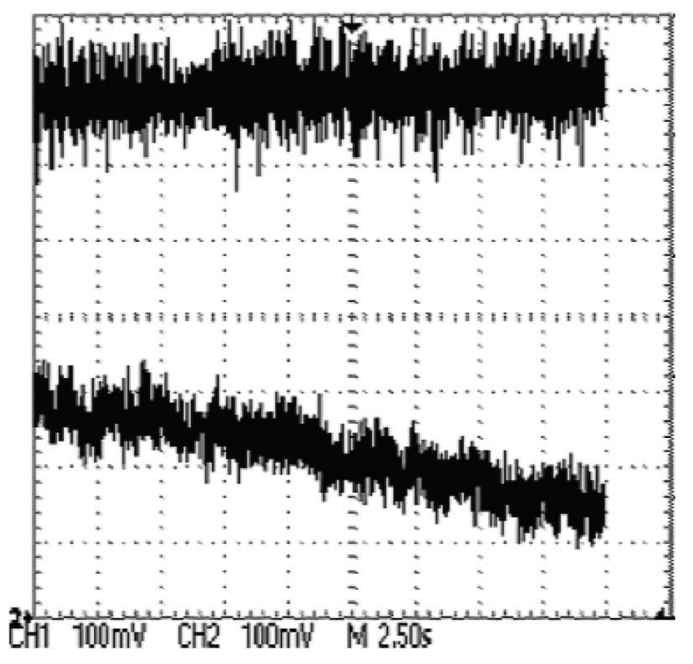

Fig. 12. Sensor response when it is in direct sunlight exposure: (top) elevation and (bottom) azimuth voltage signals.

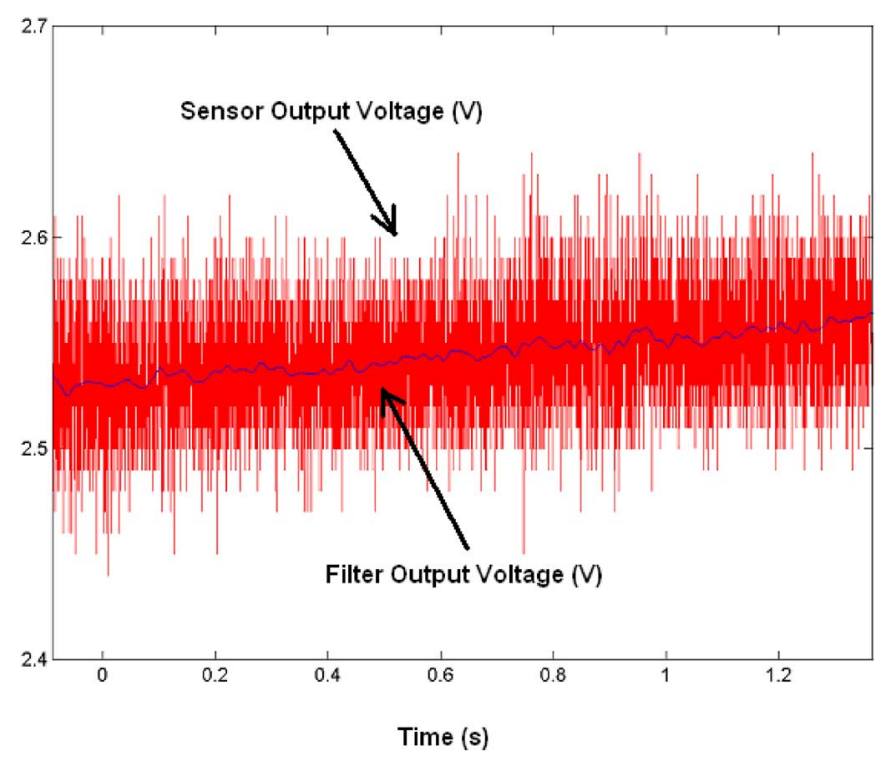

Fig. 13. Current difference signal before and after filtering.

commanded [16]. As was explained in a previous section, the differential current generated depends on the sun's radiation. Thus, the presence of a cloud changes the sensor output signal, and the microcontroller could interpret incorrectly that the sun is moving. Thus, a controller program was implemented and its simplified state diagram is presented in Fig. 14.

A Bang Bang control algorithm in each axis, which was named Sun_Tracking, was selected for normal operation mode. The value of the error signal is compared with two threshold values. When the sensor voltage is below the minimum threshold, the electric motor of the corresponding axis is activated until the signal is above the maximum threshold. These thresholds define a hysteresis band where no actuation is commanded. The threshold values can be adjusted to achieve the accuracy needed for a specific application.

The aforementioned mode is valid when the incident radiation value is significant. However, on a cloudy day, the direct radiation measured with the addition of photocurrents will be 


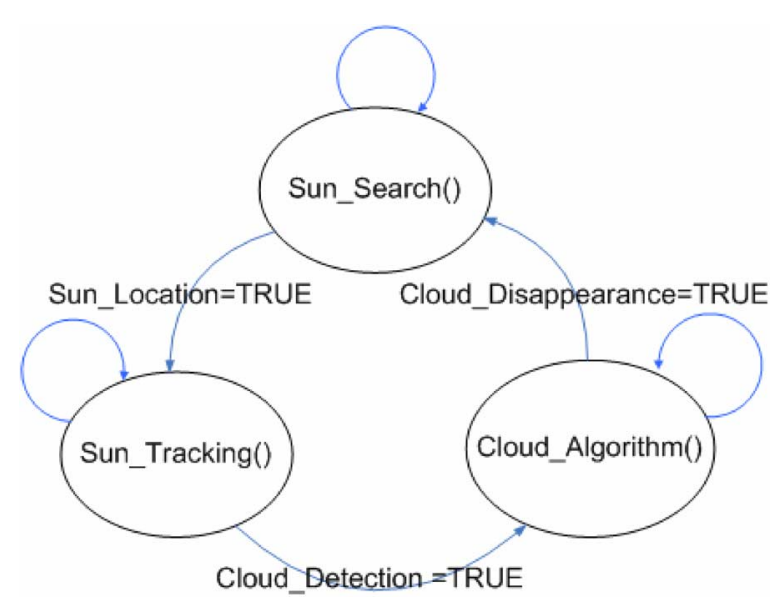

Fig. 14. State machine of the controller.

below an adaptive level when a cloud blocks the direct sunlight, so the controller will shift to the Cloud_Algorithm. In this mode, the controller repeats the last on/off duty cycle of the dc motor activations recorded during the Sun_Tracking mode. These activations extrapolate the behavior of the system during the last sunny period. If the controller is in Cloud_Algorithm mode for more than $10 \mathrm{~min}$ or the sun radiation is above a certain value, it switches to Sun_Search, a mode that waits for an increase in sun radiation and then updates the system according to the new sun position using the wide range sensors. The Sun_Search mode is also necessary in other situations, such as daybreak.

Finally, when no radiation is detected, the structure is oriented to the east until it reaches a limit switch and waits for the sunrise. The difference between a cloud crossing and nightfall is the slope of the sensor output signal.

\section{ApPlication}

In order to test the performance of the control system, it was applied to some structures to evaluate the performance of the aforementioned implementation. This control scheme was used for direct sun tracking in PV panels with mirrors [17]. However, in this paper, we focus on its use in heliostats.

In this application, two different control strategies-openloop and closed-loop schemes-are compared in order to outline their differences. These control schemes are applied to a heliostat that is controlled to reflect the energy from the sun to an absorber that is on top of a tower.

In the open-loop control (see Fig. 15), the local position of the sun is estimated using a set of equations [18] and then the control algorithm calculates the exact position of the mirror based on the relative position of the heliostat with respect to the concentrator.

In this open-loop scheme, a robust pedestal and mechanicalgeared drive are needed to minimize the deviation caused by the mirror weight and wind loads. In addition, very limited tolerances are allowed in the mechanical transmission chain to achieve a high pointing precision. Notice that the last two design requirements are antagonist, which lead to expensive mechanical structures. Furthermore, the installation requires

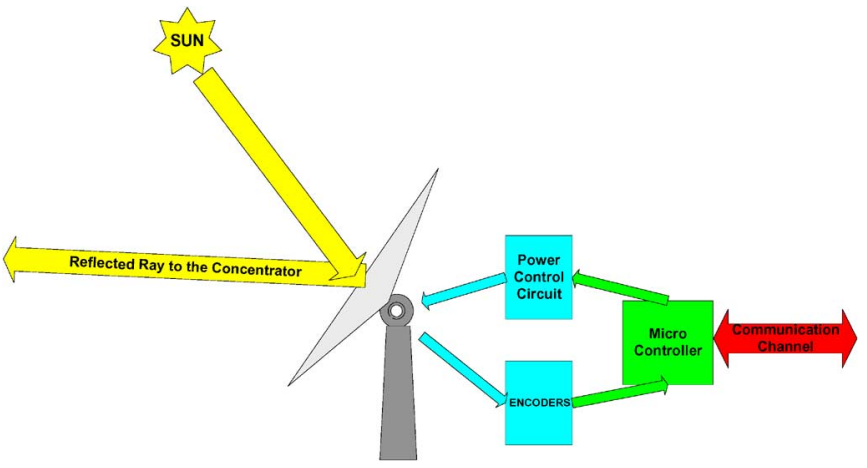

Fig. 15. Open-loop control system of a heliostat.

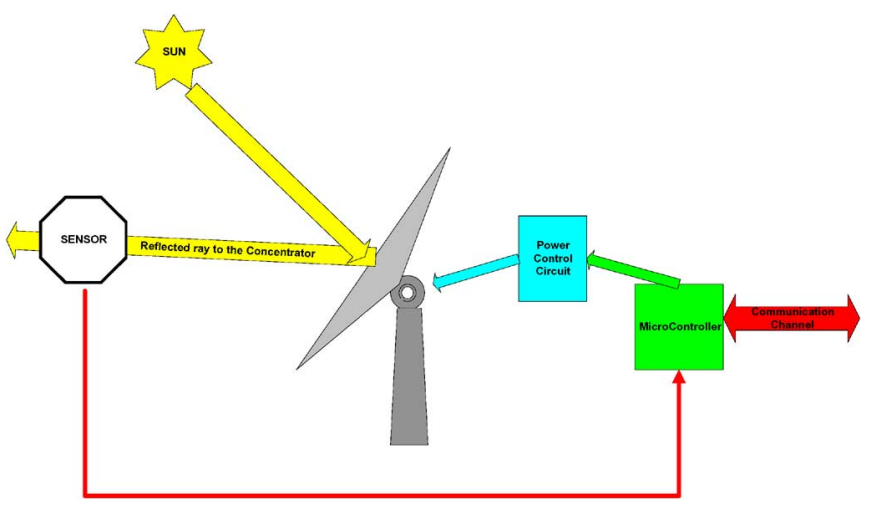

Fig. 16. Closed-loop feedback control system of a heliostat using a highaccuracy incident angle sensor.

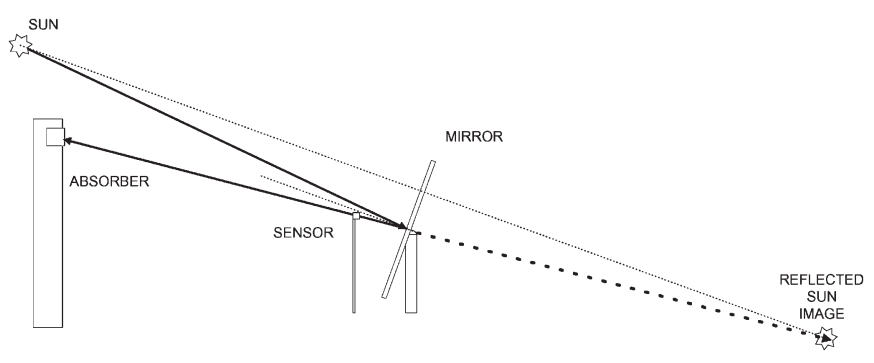

Fig. 17. Geometrical interpretation of sensor closed-loop system behavior.

very precise initial positioning with regard to the concentrating tower, and some periodic maintenance is necessary to calibrate the controller and compensate for any misalignment.

In the closed-loop feedback control, as depicted in Fig. 16, an incident angle sensor is located in the path between the heliostat and the concentrator to provide a feedback control signal. In this scheme, the sensor analyzes the reflected ray and provides an error signal generated by the misaligned orientation of the reflective surface. As the sensor is analyzing the incident angle of the reflected ray, all deviations previously described in the open-loop scheme are overcome and then the mechanical design specifications of the structure are largely relaxed.

A geometrical interpretation of the sensor behavior can be obtained in Fig. 17. The sensor placed in front of the mirror directly receives the light from a virtual reflected sun image. When the reflected ray is misaligned, a rotation angle $\theta$ in the mirror produces a shift in the virtual sun image and the same 


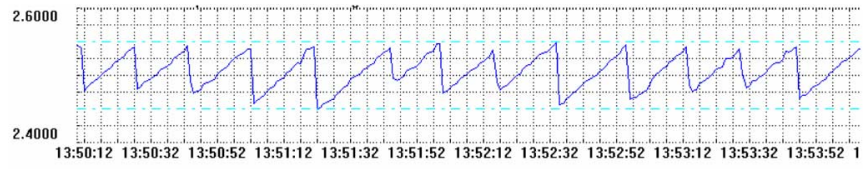

(a)

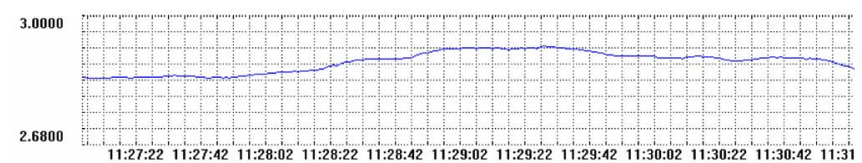

(b)

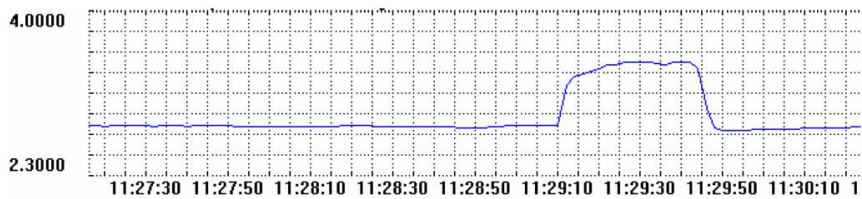

(c)

Fig. 18. Output voltage of the current difference during Sun_Tracking mode.

change in the incident angle of the reflected ray. This provides a mechanism to guarantee a correct reflected ray orientation. From the sensor's point of view, a rotation of the mirror is interpreted as a displacement of the mirrored sun. The error signal generated by the sensor can be used to rotate the mirror to align the sun with respect to the absorber.

This approach requires the sensor to be precisely positioned only once during its installation. This can be easily done either with a movable mechanical support or with an electronic offset adjustment. The sensor is calibrated using a sunray from the heliostat while the controller is in Sun_Tracking mode. The sensor is then adjusted in both axes to project the reflected sunray onto the absorber. Using this procedure, we eliminate any geometry deviation that may occur during the manufacturing and installation of the sensor.

The desired precision was achieved for this application, and only one problem was detected when the reflection of the sunray changed from one facet to another. This problem was solved by placing some sensors working in parallel and by including a comparison strategy in their error signals.

The proposed algorithm was successfully tested under different conditions (during sunny and cloudy days). Some experimental results are shown in Fig. 18. In (a), the azimuth differential voltage signal is plotted when working in Sun_Tracking mode. The saw-like waveform corresponds to the sequence of updating movements of this axis. A position update occurs approximately every $20 \mathrm{~s}$, which corresponds to less than $1 \mathrm{mrad}$ in sun movement. The upper limit voltage indicates the maximum tolerated error and defines the instant when the dc motor starts moving. Notice that the inertia of the structure forces its movement and it justifies the different voltages when the structure stops. In Fig. 18(b), the direct sun-radiation signal voltage is almost constant, and its small deviation takes place because of the variation of the direct radiation caused by a light mist. The last plot in Fig. 18 represents the variation of the direct sun-radiation signal voltage during a cloud crossing. This change switches the controller from the Sun_Tracking mode to the Cloud_Algorithm mode and vice versa.

\section{CONCLUSiON}

A novel sensor design to accurately determine the incident angle of radiation using MEMS technology has been presented. This structure consists of a set of photodiodes that are covered with a metal shield that projects a shadow over them. A detailed study of the sensor was developed, and it is worth noting that the distance from the aperture to the sensor can be regarded as a gain factor in the position sensor. As a result, a desired sensibility can be achieved by placing the aperture a sufficiently far distance from the photodiode. Other design parameters guarantee robustness against noise and fabrication tolerances. This design and its manufacturing process are patent protected [19].

A complete control algorithm was developed using this sensor. This algorithm can be used to precisely determine the position of a light source and this property has been tested in several applications, e.g., in direct tracking of PV panels and indirect control of the heliostat. In both cases, the results confirm the validity of the controller and point to a reduction in the installation and maintenance cost of these plants. The proposed control algorithm represents an important improvement in comparison with other tracking systems [20].

The use of MEMS techniques allows for a low cost and very accurate realization of this sensor. A commercial version of this device is currently being developed, including a signal conditioning circuit, an analog-to-digital converter, and an embedded microcontroller to provide a fully integrated digital system for sun-tracking applications.

\section{REFERENCES}

[1] D. Y. Goswami, S. Vijayaraghavan, S. Lu, and G. Tamm, "New and emerging developments in solar energy," Sol. Energy, vol. 76, no. 1, pp. 33-43, Jan.-Mar. 2004.

[2] E. Koutroulis and K. Kalaitzakis, "Design of a maximum power tracking system for wind-energy-conversion applications," IEEE Trans. Ind. Electron., vol. 53, no. 2, pp. 486-494, Apr. 2006.

[3] S. R. Bull, "Renewable energy today and tomorrow," Proc. IEEE, vol. 89, no. 8, pp. 1216-1226, Aug. 2001.

[4] T. M. Razykov, "Photovoltaic solar electricity: State of the art and future prospects," in Proc. 6th ICEMS, Nov. 9-11, 2003, vol. 1, pp. 297-301.

[5] E. Román, R. Alonso, P. Ibañez, S. Elorduizapatarietxe, and D. Goitia, "Intelligent PV module for grid-connected PV systems," IEEE Trans. Ind. Electron., vol. 53, no. 4, pp. 1066-1073, Aug. 2006.

[6] S. Krauter and R. Hanitsch, "Actual optical and thermal performance of PV-modules," in Proc. IEEE 1st World Conf. Photovaltic Energy Conver., Dec. 1994, pp. 734-737.

[7] H. S. Lee and M. Tomizuka, "Robust motion controller design for highaccuracy positioning systems," IEEE Trans. Ind. Electron., vol. 43, no. 1, pp. 48-55, Feb. 1996

[8] J. R. Wertz, Spacecraft Attitude Determination and Control, J. Wertz, Ed. Norwell, MA: Kluwer, 1995.

[9] R. S. Baheti and P. F. Scott, "Design of self-calibrating controllers for heliostats in a solar power plant," IEEE Trans. Autom. Control, vol. AC-25, no. 6, pp. 1091-1097, Dec. 1980.

[10] C. W. de Boom, J. A. P. Leijtens, L. M. H. V. Duivenbode, and N. van der Heiden, "Micro digital sun sensor: System in a package," in Proc. ICMEMS, NANO and Smart Syst., Aug. 25-27, 2004, pp. 322-328.

[11] R. Doraiswami and S. Price, "A robust position estimation scheme using sun sensor," IEEE Trans. Instrum. Meas., vol. 47, no. 2, pp. 595-603, Apr. 1998.

[12] J. Graeme, Photodiode Amplifiers. New York: McGraw-Hill, 1995.

[13] C. C. Liebe, "Solar compass chip," IEEE Sensors J., vol. 4, no. 6, pp. 779786, Dec. 2004.

[14] J. J. Liou, Advanced Semiconductor Device Physics and Modeling. Norwood, MA: Artech House, 1994. 
[15] J. Garcia, F. R. Palomo, A. Luque, C. Aracil, J. M. Quero, D. Carrion, F. Gamiz, P. Revilla, J. Perez-Tinao, M. Moreno, P. Robles, and L. G. Franquelo, "Reconfigurable distributed network control system for industrial plant automation," IEEE Trans. Ind. Electron., vol. 51, no. 6, pp. 1168-1180, Dec. 2004.

[16] E. C. Ker, Jr., E. M. Gulachenski, and G. A. Kern, "Cloud effects on distributed photovoltaic generation: Slow transients at the Gardner, Massachusetts photovoltaic experiment," IEEE Trans. Energy Convers., vol. 4, no. 2, pp. 184-190, Jun. 1989.

[17] P. Valera, A. Esteban, M. Carrillo, and R. Osuna, "Solar energy comparative of solar technologies for electricity production," in Proc. WCPEC-3, Osaka, Japan, May 11-18, 2003, pp. 2482-2486.

[18] J. J. Michalsky, “The astronomical almanac's algorithm for approximate solar position (1950-2050)," Sol. Energy, vol. 40, no. 3, pp. 227-235, Jun. 1988.

[19] J. M. Quero Reboul, J. García Ortega, and L.G. Franquelo. "Sensor electrónico para la medida de la posición angular de un objeto liminiscente," Spanish Patent P9901375, June 21, 1999.

[20] V. D. Rumyantsev, V. M. Andreev, N. A. Sadchikov, A. W. Bett, F. Dimroth, and G. Lange, "Experimental installations with highconcentration PV modules using III-V solar cells," presented at the Int. Conf. PV Eur.-From PV Technology Energy Solutions, Rome, Italy, 2002.

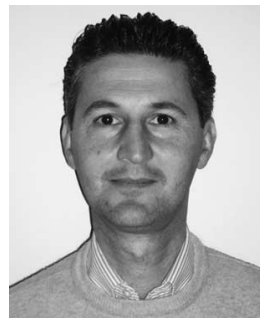

José M. Quero (M'97-SM'06) was born in Seville, Spain, in 1963. He received the M.Sc. and Ph.D. degrees in electrical engineering from the University of Seville, Seville, in 1988 and 1991, respectively.

In 1988, he joined the Department of Electronic Engineering, University of Seville, as an Assistant Professor. He became an Associate Professor in 1992 and, since 2000, he has been a Full Professor. He also teaches courses on analog and digital microelectronics, and microsystems at the Engineering School, University of Seville. He is also a Senior Researcher with the Andalusian Association for Research and Industrial Cooperation (AICIA), a nonprofit research organization. He is an Evaluator and a Reviewer for the European Commission in the Information Society Technology program. His research interests include MEMS sensors and actuators, and their application in medicine, microfluidics, and RF.

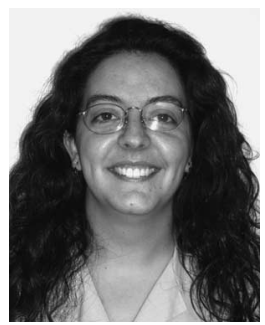

Carmen Aracil was born in Seville, Spain, in 1974. She received M.Sc. degrees in physics and electronics engineering from the University of Seville, Seville, in 2001 and 2003, respectively. She is currently working toward the Ph.D. degree in the field of BioMEMS at the same university.

In 2002, she was a Research Assistant at the University of Seville. In 2003, she joined the Department of Electronic Engineering, University of Seville, where she is currently a Research and Teaching Assistant. Her research interests include bioengineering, microfluidics, and control systems.

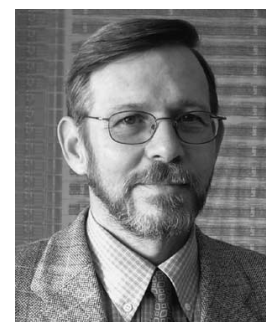

Leopoldo G. Franquelo (M'85-SM'96-F'05) was born in Málaga, Spain. He received the M.Sc. and $\mathrm{Ph} . \mathrm{D}$. degrees in electrical engineering from the University of Seville, Seville, Spain, in 1977 and 1980, respectively.

In 1978, he joined the University of Seville as a Research Assistant. He became an Associate Professor in 1982 and a Professor in 1986. From 1998 to 2005, he was a Director in the Department of Electronic Engineering, University of Seville. He is currently responsible for the Ph.D. courses in the same department. His technical interests started in 1978 with microprocessor industrial electronics applications, evolving to electronics power applications, and in the 1990s, to application-specific ICs design for the control of power converters. He is leading a large research and teaching team in Spain. In the last five years, his group activity can be summarized as: 33 publications in international journals, 160 in international proceedings, ten patents, advisor for ten Ph.D. dissertations, and $84 \mathrm{R} \& \mathrm{D}$ projects. His research team has been awarded with the "Excellence Status" by the Regional Government. His current research interests include modulation techniques for multilevel inverters and their application to power electronic systems for renewable energy systems.

Dr. Franquelo has been a Vice-President of the IEEE Industrial Electronics Society Spanish Chapter (2002-2003), a Member-at-Large of the IEEE Industrial Electronics Society AdCom (2002-2003), and a Vice-President for Conferences of the IEEE Industrial Electronics Society since 2004.

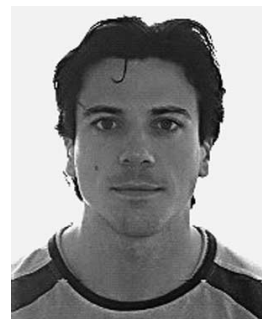

Jordi Ricart was born in Sabadell, Spain, in 1976 He received the M.Sc. degree in ingeniería electrónica from the Universitat Politècnica de Catalunya (UPC), Barcelona, Spain, in 2004, where he is currently working toward the Ph.D. degree.

$\mathrm{He}$ is also currently a Research Assistant in the Semiconductor Devices Group, Departamento de Ingeniería Electrónica de la Escuela Técnica Superior de Ingeniería de Telecomunicación. He has worked in photovoltaic processes and thermal flowmeter fabrication. His research interests also include the design of specific circuits for MEMS, such as oscillators.

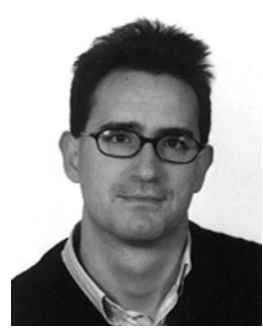

Pablo R. Ortega was born in Barcelona, Spain, in 1966. He received the M.Sc. and Ph.D. degrees in telecommunication engineering from the Universitat Politècnica de Catalunya (UPC), Barcelona, in 1991 and 2000, respectively. His Ph.D. dissertation research work focused on the development of new packaging strategies for microphotovoltaic systems.

$\mathrm{He}$ is currently an Assistant Professor with the Semiconductor Devices Group, Departamento de Ingeniería Electrónica de la Escuela Técnica Superior de Ingeniería de Telecomunicación, UPC. He is currently involved with design, fabrication, characterization, and simulation of photovoltaic devices, as well as studying new applications for photovoltaic energy conversion.

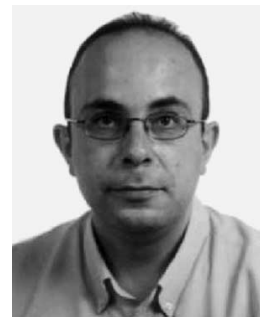

Manuel Domínguez (M'99) received the M.Sc. and $\mathrm{Ph} . \mathrm{D}$. degrees in electronic engineering from the Universitat Politècnica de Catalunya (UPC), Barcelona, Spain, in 1994 and 1997, respectively, and the M.Sc. degree (with honors) in mathematics from the Universidad Nacional de Educación a Distancia, Madrid, Spain, in 2005.

Since 1994, he has been with the Departamento de Ingeniería Electrónica de la Escuela Técnica Superior de Ingeniería de Telecomunicación, UPC, as an Associate Professor. His research interests include the design and development of MEMS sensors and actuators, cMUTs, sigma-delta modulation applied to MEMS, and nonlinear circuits for MEMS. 


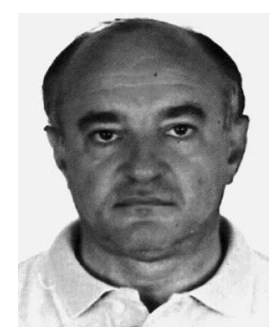

Luis M. Castañer (S'75-M'77-SM'92) received the Ingeniero Superior de Telecomunicación degree from the Universidad Politécnica de Madrid, Madrid, Spain, in 1971, the Diplôme d'Etudes Approfondies (D.E.A.) degree in spatial physics and the DocteurIngenieur degree from the Université Paul Sabatier, Toulouse, France, in 1972 and 1973, respectively, and the Doctor Ingeniero de Telecomunicación degree from the Universitat Politècnica de Catalunya (UPC), Barcelona, Spain.

$\mathrm{He}$ is currently a Professor in the Departamento de Ingeniería Electrónica de la Escuela Técnica Superior de Ingeniería de Telecomunicación, UPC. He has also been the Dean of E.T.S.I. Telecomunicación and the Head of the Departamento de Ingeniería Electrónica. He has held several positions in research agencies and committees: Representative in the Comite de Gestión y Coordinación on nonnuclear energies, DGXII Commission of the EU, Head of the Programa Nacional de Tecnologías de la Información y Comunicaciones of the CICYT (1992-1994), and Coordinator of the Technology Foresight at the Agencia Nacional de Evaluación y Prospectiva. $\mathrm{He}$ has contributed to semiconductor device research, covering solar cells in various aspects - technology of CIS and space degradation of silicon and GaAs devices - and to the theory and technology of advanced bipolar transistors with polysilicon emitters and their application to high-efficiency silicon solar cells, in particular, to the emitter resistance of these devices. He has also contributed to the design, simulation, and monitoring of photovoltaic power plants and systems. He is active in the microsystems technology area, working on flow sensors, power devices for MEMS, and electrostatic actuators.

Dr. Castañer is a member of the Association and Charter of Telecommunication Engineers in Spain and of the Spanish Engineering Academy.

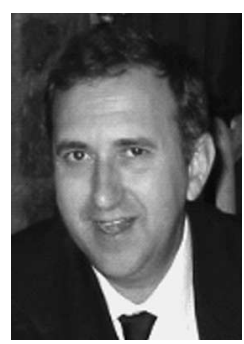

Rafael Osuna received the M.Sc. degree in electrical engineering from the Universidad de Sevilla, Seville, Spain and the M.B.A. degree from the Universidad de Deusto, Bilbao, Spain.

His professional activity is linked to Abengoa, where he has managed engineering projects in power electronics and control. He has developed power supplies for superconductor magnets for the Centre European per la Reserche Nucleare (CERN). In 1995, he became responsible for the Toledo-PV, a 1-MWe photovoltaic plant. In 1999, being responsible for the Solar Group in Inabensa, he led the construction of PS10, a solarconcentrating power plant using heliostats. Since 2002, he has been the CEO of Sanlúcar Solar Solúcar, Seville. 